\title{
Estimation of a planetary magnetic field using a reduced magnetohydrodynamic model
}

\author{
Christian Nabert $^{1}$, Daniel Heyner ${ }^{1}$, and Karl-Heinz Glassmeier ${ }^{1,2}$ \\ ${ }^{1}$ Institut für Geophysik und extraterrestrische Physik, Technische Universität Braunschweig, \\ Mendelssohnstr. 3, 38106 Braunschweig, Germany \\ ${ }^{2}$ Max-Planck-Institut für Sonnensystemforschung, Justus-von-Liebig-Weg 3, 37077 Göttingen, Germany \\ Correspondence to: Christian Nabert (c.nabert@tu-bs.de)
}

Received: 6 December 2016 - Revised: 3 March 2017 - Accepted: 6 March 2017 - Published: 22 March 2017

\begin{abstract}
Knowledge of planetary magnetic fields provides deep insights into the structure and dynamics of planets. Due to the interaction of a planet with the solar wind plasma, a rather complex magnetic environment is generated. The situation at planet Mercury is an example of the complexities occurring as this planet's field is rather weak and the magnetosphere rather small. New methods are presented to separate interior and exterior magnetic field contributions which are based on a dynamic inversion approach using a reduced magnetohydrodynamic (MHD) model and time-varying spacecraft observations. The methods select different data such as bow shock location information or magnetosheath magnetic field data. Our investigations are carried out in preparation for the upcoming dual-spacecraft BepiColombo mission set out to precisely estimate Mercury's intrinsic magnetic field. To validate our new approaches, we use THEMIS magnetosheath observations to estimate the known terrestrial dipole moment. The terrestrial magnetosheath provides observations from a strongly disturbed magnetic environment, comparable to the situation at Mercury. Statistical and systematic errors are considered and their dependence on the selected data sets are examined. Including time-dependent upstream solar wind variations rather than averaged conditions significantly reduces the statistical error of the estimation. Taking the entire magnetosheath data along the spacecraft's trajectory instead of only the bow shock location into account further improves accuracy of the estimated dipole moment.
\end{abstract}

Keywords. Geomagnetism and paleomagnetism (general or miscellaneous) - magnetospheric physics (magnetosheath; solar wind-magnetosphere interactions)

\section{Introduction}

The interaction of a planetary magnetic field with the solar wind strongly modifies the magnetic field environment around the planet. If in situ spacecraft data are used to estimate the planetary magnetic field, this interaction needs to be taken into account. This is of particular importance for the upcoming two-spacecraft mission BepiColombo (Benkhoff et al., 2010) to planet Mercury because the Hermean magnetosphere is small and very dynamic. The average magnetopause distance at Mercury observed by the MESSENGER (Mercury Surface, Space Environment, Geochemistry and Ranging) mission (Solomon et al., 2001) is about $1.45 R_{\mathrm{M}}$ and the bow shock distance is $1.89 R_{\mathrm{M}}$, with $R_{\mathrm{M}}=2440 \mathrm{~km}$ as planetary radius (Winslow et al., 2013). Consequently, the currents of the interaction influence the magnetic field distribution along the entire orbit of a spacecraft such as the BepiColombo planetary orbiter at Mercury. The two BepiColombo spacecraft, the Mercury Planetary Orbiter (MPO) and the Mercury Magnetospheric Orbiter (MMO), investigate Mercury's environment on polar orbits (see http://sci. esa.int/). The MPO will travel close to the surface of Mercury with a period of $2.3 \mathrm{~h}$, a periapsis of $1.2 R_{\mathrm{M}}$, and an apoapsis of $1.6 R_{\mathrm{M}}$ with respect to Mercury's center. The MMO will travel farther out with a period of $9.2 \mathrm{~h}$, a periapsis of $1.2 R_{\mathrm{M}}$, and an apoapsis of $5.8 R_{\mathrm{M}}$. At the aphelion of Mercury, the periapsides are the subsolar point, and at perihelion, the apoapsides are the subsolar point. Thus, especially around perihelion, the MMO will spend most of the time in the solar wind and crosses the bow shock twice on each orbit. Note that the MPO will usually not cross the bow shock and observes the interaction region close to the planet. 
In this kind of dynamic environment interspersed with local electrical currents, the classic Gauss algorithm fails to separate internal and external magnetic fields in a classical spherical harmonic expansion (Gauss, 1839; Olsen et al., 2010; Glassmeier and Tsurutani, 2014). In addition, spacecraft missions provide only incomplete spatial coverage. For example, the MESSENGER mission only provided observations close to the Hermean surface in the northern hemisphere. This leads to a systematic correlation of Gauss coefficients. Therefore, more elaborate approaches to determine the internal field must be sought often such as the methods presented here. Accurate internal field determination is crucial, for example, for the identification of suitable dynamo models which could explain the enigmatic Hermean planetary magnetic field (e.g., Heimpel et al., 2005; Stanley et al., 2005; Christensen, 2006; Glassmeier et al., 2007; Heyner et al., 2011; Wicht and Heyner, 2014).

We investigate various methods to estimate a planetary magnetic field from in situ spacecraft observations. Previous approaches consider the interaction of Mercury's magnetic field with the solar wind using empirical models. For example, Korth et al. (2004) used a scaled Tsyganenko-96 model of the Earth's magnetosphere (Tsyganenko, 1995, 1996) to include current systems of the interaction at Mercury. Another empirical model used at Mercury is the paraboloid magnetosphere model introduced by Alexeev et al. (2010). Such a model was also used by Johnson et al. (2012) to estimate Mercury's magnetic field with data from MESSENGER. Further improvement of modeling the magnetosphere was done by Korth et al. (2015), including parametric models of magnetopause and cross-tail currents. In general, empirical models either contain many parameters which also depend on solar wind conditions or use several assumptions such as the prescription of shape and location of interaction currents which reduce the number of parameters to be estimated from data.

Here, we consider two different approaches employing an magnetohydrodynamic (MHD) model to compute the interaction depending on the varying solar wind conditions. For an efficient calculation, we use a reduced MHD model presented by Nabert et al. (2013) which is derived from the MHD equations using series expansion techniques. Further, the reduced model is less sensitive to numerical errors. The reduced models are suitable for detailed investigations of the estimation procedure of the planetary magnetic field and the related errors. Such a reduced model was successfully applied to reconstruct the solar wind conditions from terrestrial magnetosheath data (Nabert et al., 2015). Spacecraft data from the interaction region and the known planetary magnetic field of the Earth were used to estimate the solar wind conditions at the subsolar point of the bow shock. Here, this approach is reversed: the spacecraft data together with solar wind conditions are used to estimate the planetary magnetic field.
We consider terrestrial THEMIS data to reconstruct the well-known planetary magnetic field of the Earth as a test case for the more challenging situation at Mercury in preparation for the BepiColombo mission. However, with respect to the strongly modified magnetic field environment due to the solar wind at Mercury, we choose THEMIS data from the magnetosheath. In this region, the measured magnetic field at the Earth is strongly influenced by the interaction with the solar wind comparable to the situation at Mercury.

With the reduced MHD model, different procedures can be applied to obtain the planetary magnetic moment and are investigated with respect to systematic and statistical errors. A first method, suitable also for single-spacecraft missions, considers observations from a spacecraft crossing the bow shock which measures the solar wind conditions on the sunward side of the shock. Then, Earth's dipole moment can be directly calculated with analytical expressions of the shock's distance in the reduced MHD model. At Mercury, this approach is applicable for the BepiColombo mission using the bow shock observations of the MMO around perihelion. Note that, in general, the quality of solar wind data of the MESSENGER mission at Mercury is not sufficient to apply this method presented here (Raines et al., 2011, 2013). A second approach is to use spacecraft data from the entire magnetosheath passage along the spacecraft's trajectory with time-dependent solar wind observations from another spacecraft. Here, we take magnetosheath data at Earth into account to consider a highly disturbed environment similar to Mercury. Note that, in general, this approach is not restricted to magnetosheath data. The magnetospheric magnetic field of the model is determined by the Biot-Savart law using the magnetosheath current density and can be compared to magnetospheric observations. To improve precision of such an extension, further current contributions can be included from different models as well. The approach can be applied whenever the MPO provides solar wind data.

\section{The reduced MHD model by Nabert et al. (2013)}

The reduced MHD model by Nabert et al. (2013) provides solutions of the ideal MHD equations for the mass density $\rho$, the plasma velocity $\boldsymbol{v}$, the gas pressure $p$, and the magnetic field $\boldsymbol{B}$ assuming a quasi-stationary situation. In the magnetosheath, the ideal, stationary MHD equations are

$$
\begin{aligned}
0 & =\nabla \cdot(\rho \boldsymbol{v}), \\
0 & =\rho(\boldsymbol{v} \cdot \nabla) \boldsymbol{v}+\nabla p-\frac{1}{\mu_{0}}(\nabla \times \boldsymbol{B}) \times \boldsymbol{B}, \\
0 & =\nabla \times(\boldsymbol{v} \times \boldsymbol{B}), \\
0 & =\nabla \cdot \boldsymbol{B}, \\
p & =k_{\mathrm{p}} \rho^{\gamma},
\end{aligned}
$$

with the constant of proportionality $k_{\mathrm{p}}$ of the adiabatic law (5) and the vacuum permeability $\mu_{0}=4 \pi \cdot 10^{-7} \mathrm{~N} / \mathrm{A}^{2}$. The 
solution of the MHD equations can be expressed in vector notation:

$\boldsymbol{u}:=\left(\rho, v_{x}, v_{y}, v_{z}, p, B_{x}, B_{y}, B_{z}\right)^{T}$.

A Cartesian coordinate system with the solar wind along the $x$ direction and perpendicular directions $y$ and $z$ is considered. The origin of the coordinate system is located at the bow shock. The general idea of the approach by Nabert et al. (2013) is to derive reduced MHD models of the interaction with series expansions of the physical quantities. Each physical quantity $u^{n}(x, y, z)$, where $n$ labels the component of the vector $\boldsymbol{u}$ according to Eq. (6), is expanded by a Taylor series with respect to the $y$ and $z$ directions:

$u^{n}(x, y, z)=\sum_{j=0}^{N_{\mathrm{S}}+1} \sum_{k=0}^{N_{\mathrm{S}}+1} u_{j k}^{n}(x) y^{j} z^{k}$

Here, $\left(N_{\mathrm{S}}+1\right)$ denotes the expansion order and $u_{j k}^{n}(x)$ the coefficient functions.

To simplify the considerations, we assume a quasistationary situation with a solar wind magnetic field and a planetary dipole moment along the $z$ direction. Then, the dipole approximation of the Earth's planetary magnetic field is

$B_{\mathrm{E}, x}=\frac{3 z\left(x_{\mathrm{E}}-x\right)}{r^{5}} M$,

$B_{\mathrm{E}, y}=\frac{3 y z}{r^{5}} M$,

$B_{\mathrm{E}, z}=-\frac{-2 z^{2}+\left(x_{\mathrm{E}}-x\right)^{2}+y^{2}}{r^{5}} M$,

with distance $r:=\left(\left(x_{\mathrm{E}}-x\right)^{2}+y^{2}+z^{2}\right)^{0.5}$, dipole moment $M$, and the distance of the bow shock to the Earth's center $x_{\mathrm{E}}$ along the $x$ axis. The largest quadrupole contribution to the magnetic field at about $10 R_{\mathrm{E}}$, an approximation of the subsolar magnetopause distance at Earth, is less than $1 \%$, where $R_{\mathrm{E}}=6371 \mathrm{~km}$. Therefore, we neglect any quadrupole contributions. It should be noted that this is a situation very different from that one at planet Mercury. However, it seems a valid approximation to introduce an offset dipole to include Mercury's quadrupole contributions (Johnson et al., 2012). The dipole moment representation and solar wind magnetic field perpendicular to the solar wind direction requires certain symmetries of the physical quantities, which leads to vanishing coefficient functions (Nabert et al., 2013). A symmetric quantity $u^{n}(x, y, z)$ to the $x y$ plane satisfies $u^{n}(x, y,-z)=u^{n}(x, y, z)$, an anti-symmetric quantity satisfies $u^{n}(x, y,-z)=-u^{n}(x, y, z)$ and analogous with respect to the $x z$ plane. In particular, $\rho, v_{x}, p$, and $B_{z}$ are symmetric whereas $B_{y}$ is anti-symmetric with respect to both planes. Further, $B_{x}$ and $v_{z}$ are anti-symmetric to the $x y$ plane and symmetric to the $x z$ plane. In contrast, $v_{y}$ is symmetric to the $x y$ plane and anti-symmetric to the $x z$ plane.
Similar to the physical quantities, the bow shock and magnetopause geometry are expanded into Taylor series with respect to the $y$ and $z$ direction. We restrict our considerations to the situation at Earth and choose $N_{\mathrm{S}}=0$. The latter assumption restricts the model to the vicinity of the $x$ axis. The origin of the coordinate system coincides with the subsolar point of the bow shock. Further, bow shock and magnetopause can be approximated by series expansions to the $y$ and $z$ direction up to the second order. Taking into account the symmetries, the shock geometry is given by

$x=c_{\mathrm{BS}, y} y^{2}+c_{\mathrm{BS}, z} z^{2}$,

where the coefficients $c_{\mathrm{BS}, y}$ and $c_{\mathrm{BS}, z}$ determine curvatures of the shock. Similarly, the magnetopause parametrization is given by

$x=x_{\mathrm{MS}}+c_{\mathrm{MP}, y} y^{2}+c_{\mathrm{MP}, z} z^{2}$,

where $x_{\mathrm{MS}}$ is the distance of the magnetopause to the bow shock along the $x$ axis, and $c_{\mathrm{MP}, y}$ and $c_{\mathrm{MP}, z}$ are magnetopause curvature parameters. The expansions of the bow shock and the magnetopause are used to introduce a new coordinate $\tilde{x}$ which adjusts the coordinate system to the magnetosheath geometry. The new coordinate $\widetilde{x}$ is defined by

$\tilde{x}=x-\left(c_{\mathrm{BS}, y}+\Delta c_{y} \frac{\tilde{x}}{x_{\mathrm{MS}}}\right) y^{2}-\left(c_{\mathrm{BS}, z}+\Delta c_{z} \frac{\tilde{x}}{x_{\mathrm{MS}}}\right) z^{2}$,

with $\Delta c_{y}:=c_{\mathrm{MP}, y}-c_{\mathrm{BS}, y}$ and $\Delta c_{z}:=c_{\mathrm{MP}, z}-c_{\mathrm{BS}, z}$. Note that $\tilde{x}=0$ gives the bow shock parametrization (11) and $\tilde{x}=x_{\mathrm{MS}}$ gives the magnetopause parametrization (12). Approximative analytical expressions for the curvature parameters are determined by $c_{\mathrm{MP}, y}=2 /\left(5 \Delta x_{\mathrm{MP}}\right), c_{\mathrm{MP}, z}=$ $1 /\left(2 \Delta x_{\mathrm{MP}}\right), c_{\mathrm{BS}, y}=2 /\left(5 \Delta x_{\mathrm{BS}}\right)$, and $c_{\mathrm{BS}, z}=1 /\left(2 \Delta x_{\mathrm{BS}}\right)$, where $\Delta x_{\mathrm{BS}}=\left|x_{\mathrm{E}}\right|$ and $\Delta x_{\mathrm{MP}}$ denotes the distance of the magnetopause to the Earth's center (Nabert et al., 2013).

The coordinate $x$ is replaced by the new coordinate $\tilde{x}$ in expansion (7). Then, the series expansion fits the magnetosheath geometry which leads to faster convergence of the series expansions. For $N_{\mathrm{S}}=0$ and taking into account the symmetries of the situation considered as described above, series expansion (7) simplifies to

$\rho(x, y, z)=\rho_{0}(\widetilde{x})$

$\boldsymbol{v}(x, y, z)=\left(v_{x 0}(\widetilde{x}), v_{y 10}(\widetilde{x}) y, v_{z 01}(\widetilde{x}) z\right)^{T}$,

$p(x, y, z)=p_{0}(\widetilde{x})$,

$\boldsymbol{B}(x, y, z)=\left(B_{x 01}(\widetilde{x}) z, B_{y 11}(\widetilde{x}) y z, B_{z 0}(\widetilde{x})\right)^{T}$,

where the new coordinate $\tilde{x}$ is used and terms of zeroth order in $y$ and $z$ are labeled by 0 instead of 00 .

Substituting this ansatz (14)-(17) into the MHD equations (1)-(5) and equating coefficients of the lowest order, i.e., $y=$ $z=0$, a system of ordinary differential equations is obtained:

$\left(\rho_{0} v_{x 0}\right)^{\prime}+\rho_{0}\left(v_{y 10}+v_{z 01}\right)=0$, 
$\left(B_{z 0} v_{x 0}\right)^{\prime}+B_{z 0} v_{y 10}=0$,

$\rho_{0} v_{x 0} v_{x 0}^{\prime}+p_{0}^{\prime}+\frac{B_{z 0} B_{z 0}^{\prime}-B_{x 01} B_{z 0}}{\mu_{0}}=0$,

$p_{0}=k \rho_{0}^{\gamma}$.

Derivatives with respect to $\tilde{x}$ are marked by a prime, e.g., $p_{0}^{\prime}=\partial_{\tilde{x}} p_{0}$.

The coefficients of the highest order, i.e., $v_{y 10}, v_{z 01}$, and $B_{x 01}$, are assumed to be constant to close the system of ordinary differential Eqs. (18)-(21). The values of all coefficients directly behind the bow shock at $\tilde{x}=0$ are related to the solar wind by solving the Rankine-Hugoniot conditions with respect to the shock geometry (e.g., Petrinec and Russell, 1997). The solar wind conditions are denoted by $N_{\mathrm{SW}}$ for the density, $v_{\mathrm{SW}}$ for the velocity, $B_{\mathrm{SW}}$ for the magnetic field, and $p_{\mathrm{SW}}$ for the gas pressure. The expansion coefficients of the bow shock parametrization (11) and the magnetopause parametrization (12) are determined by inner boundary conditions related to the planetary magnetic field (8)(10). The magnetopause is considered as a rigid boundary and the plasma flow needs to be tangential to this boundary. Further, the magnetopause holds a modified pressure balance according to Mead and Beard (1964). A detailed derivation of all boundary conditions and higher-order equations can be found in Nabert et al. (2013). System (18)-(21) with the corresponding boundary conditions is referred to as the reduced MHD model of zeroth order.

This model presented is restricted to solar wind magnetic fields along the $z$ direction. However, a $y$ component can be taken approximately into account by substituting $B_{z} \leftarrow$ $\left(B_{z}+B_{y}\right)^{0.5}$ as shown in Nabert et al. (2015). Thus, the magnetic field within the $y z$ plane is chosen as a new $z$ component of the magnetic field. Note that effects of a magnetic field's $x$ component in the solar wind does not contribute to the zeroth-order solution (Nabert et al., 2015).

Further, we briefly summarize the important relations of an approximative solution of the zeroth-order model used in this study presented in Nabert et al. (2013) as well. The subsolar magnetopause distance to the Earth's center $\Delta x_{\mathrm{MP}}$ can be expressed by

$\Delta x_{\mathrm{MP}}=a_{\mathrm{MP}} M^{\frac{1}{3}}$

where $a_{\mathrm{MP}}$ depends on the solar wind conditions and is defined by

$a_{\mathrm{MP}}:=\left(\frac{f^{2}}{2 \mu_{0} k_{\mathrm{P}} \rho_{\mathrm{SW}} v_{\mathrm{SW}}^{2}}\right)^{\frac{1}{6}}$.

As shown by Mead and Beard (1964) $f \approx 2.44$ and according to Kivelson and Russell (1995) $k_{\mathrm{P}} \approx 0.89$ hold for a broad range of solar wind conditions. The thickness of the magnetosheath along the $\widetilde{x}$ direction is given by

$x_{\mathrm{MS}}=a_{\mathrm{MS}} a_{\mathrm{MP}} M^{\frac{1}{3}}$, with the solar wind dependent factor

$a_{\mathrm{MS}}:=\frac{1}{\left(0.8+m_{\mathrm{BS}}\right)\left(g_{\mathrm{v}}-1\right)-1}$.

Here, $g_{\mathrm{v}}$ denotes the ratio of the solar wind velocity to the post-shock velocity and $m_{\mathrm{BS}}$ measures the solar wind magnetization:

$m_{\mathrm{BS}}:=1-\frac{1}{1+\frac{\mu_{0} \gamma p_{0}(0)}{B_{z 0}^{2}(0)}}$.

Note that $g_{\mathrm{v}}$ and $m_{\mathrm{BS}}$ are determined using the RankineHugoniot conditions to obtain an analytical solution for the situation considered (e.g., Siscoe, 1983). The expression $a_{\mathrm{MS}}$ was derived as part of an analytical solution of the reduced MHD model in Nabert et al. (2013). The factor $a_{\mathrm{MS}}$ describes magnetosheath variations apart from variations in the magnetopause distance. For example, it contains the magnetosheath broadening due to a magnetic pile-up of solar wind magnetic field.

Gas pressure and temperature are second-order moments of the velocity distribution function which are difficult to determine precisely from spacecraft data McFadden et al. (2008). Thus, we use a cold plasma approximation for our approaches - i.e., the limit of a vanishing solar wind gas pressure is considered.

Although the assumptions of the model are usually valid at Earth and Mercury, our approach can be generalized. Instead of considering only a dipole moment, higher-order moments can be included as well, modifying Eqs. (8)-(10). Then, a more general system of ordinary differential equations is necessary which is derived using Eq. (7) instead of ansatz (14)(17).

\section{Estimating the dipole moment}

\subsection{Using the bow shock location}

For a single-spacecraft mission such as MESSENGER, usually no solar wind data are available while the spacecraft is crossing the interaction region. Only at the bow shock can the solar wind conditions often be extracted from the spacecraft data directly in front of the shock. Within the scope of ideal MHD, unperturbed solar wind reaches the bow shock and is decelerated at the infinitesimal thin shock. This approximation is usually valid at the bow shock of Earth and Mercury because the shock's thickness is much smaller than the shock's subsolar distance. Then, the solar wind information and bow shock location can be used to estimate planetary magnetic field parameters with an MHD model of the interaction.

We consider the subsolar bow shock distance resulting from the analytical approximation of the reduced MHD model of the magnetosheath solution by Nabert et al. (2013). 
The subsolar bow shock distance $\Delta x_{\mathrm{BS}}$, the sum of the magnetopause distance (Eq. 22) and the magnetosheath thickness (Eq. 24), is given by

$\Delta x_{\mathrm{BS}}=\left(1+a_{\mathrm{MS}}\right) a_{\mathrm{MP}} M^{\frac{1}{3}}$.

The coefficients $a_{\mathrm{MP}}$ and $a_{\mathrm{MS}}$ are determined by the solar wind conditions at the bow shock as seen in Eqs. (23) and (25) using Rankine-Hugoniot relations.

The terrestrial dipole moment is calculated with THEMIS data of the THC spacecraft in GSM coordinates. The data need to be transferred into the coordinate system of the model. Therefore, the GSM coordinates are first rotated around the $z$ axis, and then around the $y$ axis to align the solar wind velocity vector with the $x$ axis. Further, the origin is transferred from the Earth's center to the bow shock by $x \rightarrow \Delta x_{\mathrm{BS}}+x$. Although the $x$ axis of the transformed GSM coordinates is aligned with the $x$ axis of the model coordinates, the $z$ axis is not necessarily aligned with dipole moment as in the model. Thus, the projection of the dipole moment onto the $z$ axis is determined. Assuming a solar wind within the $x y$ plane of the GSM coordinates, the strength of the Earth's dipole moment in the model varies in the range of its $z$ component and its magnitude in geographic coordinates. Then, using the IGRF (Finlay et al., 2010), the dipole moment $M$ varies between

$-7.74 \times 10^{15} \mathrm{Tm}^{3}<M \leq-7.63 \times 10^{15} \mathrm{Tm}^{3}$.

Thus, the accuracy of an estimation of the dipole moment using the MHD model presented in Sect. 2 is limited to this range.

With respect to the new transformed coordinates, the measured bow shock is located at $x_{\mathrm{SC} / \mathrm{BS}}$ in the $x$ direction, at $y_{\mathrm{SC} / \mathrm{BS}}$ in the $y$ direction, and at $z_{\mathrm{SC} / \mathrm{BS}}$ in the $z$ direction. Instead of $x_{\mathrm{SC} / \mathrm{BS}}$, which requires the subsolar bow shock position known to be calculated from observations in GSM coordinates, the distance along the $x$ direction from the Earth's center $\Delta x_{\mathrm{SC} / \mathrm{BS}}$ is introduced which can be easily determined. If the bow shock crossing occurs at a location off the stagnation streamline that is beside the $x$ axis, the geometry of the shock according to Eq. (11) is taken into account to calculate the subsolar bow shock distance in Eq. (27). Using the analytical expression for the curvature parameters, the bow shock parametrization (11) is

$x=\frac{2}{5 \Delta x_{\mathrm{BS}}} y^{2}+\frac{1}{2 \Delta x_{\mathrm{BS}}} z^{2}$.

Then, the subsolar bow shock distance is related to the measured location by

$\Delta x_{\mathrm{BS}}=\frac{\Delta x_{\mathrm{SC} / \mathrm{BS}}}{2}+\sqrt{\frac{\Delta x_{\mathrm{SC} / \mathrm{BS}}^{2}}{4}+\frac{2 y_{\mathrm{SC} / \mathrm{BS}}^{2}}{5}+\frac{z_{\mathrm{SC} / \mathrm{BS}}^{2}}{2}}$.

Solar wind conditions need to be known in addition to the subsolar bow shock distance in order to calculate the dipole moment with Eq. (27). Mean values of 5 min of THC data in front of the shock transition are used to determine the solar wind conditions at the shock transition. The data are taken with a 10 min time gap to the shock because MHD theory is only approximately valid and solar wind plasma can be affected even in front of the shock. Ten minutes correspond to a spatial distance of about $600 \mathrm{~km}$ because of the spacecraft's velocity of about $1 \mathrm{~km} \mathrm{~s}^{-1}$

We investigate 11 bow shock transitions close to the subsolar point on 8 orbits of the THC spacecraft between $24 \mathrm{Au}-$ gust and 6 September 2008. During this time interval, magnetosheath transitions near the stagnation streamline can be observed as discussed below. The data are presented in Table 1 . Due to solar wind variations, multiple bow shock transitions were observed on 2, 4 and 6 September 2008. Note that sufficient solar wind data are available between two bow shock crossings to obtain individual solar wind conditions for each crossing. Each observed bow shock transition is used to calculate the dipole moment $M$ with Eq. (27). Values for multiple shock crossings on an orbit are combined to a single mean value. The corresponding relative error $\Delta M$ of each calculation is less than $19 \%$ with respect to the true value of the Earth's dipole moment according to Eq. (28). The mean value of the computed dipole moments from the 8 orbits is $M=-7.9 \times 10^{15} \mathrm{Tm}^{3}$ with a standard deviation of $0.8 \times 10^{15} \mathrm{Tm}^{3}$. The error of the mean value differs less than $4 \%$ from the true value of the $z$ component and less than $2 \%$ from the magnitude.

During the period considered, the THC spacecraft's distance to the $x$ axis is less than $5 R_{\mathrm{E}}$, except for the transitions on 24 and 25 August 2008. The subsolar bow shock distance $\Delta x_{\mathrm{BS}}$ is less than $1.1 R_{\mathrm{E}}$ away from the observed shock distance beside the $x$ axis $\Delta x_{\mathrm{SC} / \mathrm{BS}}$ according to Eq. (30). A model error of the bow shock distance can assumed to be much smaller than this correction; for example, we might choose an error of less than $0.2 R_{\mathrm{E}}$. The $z$ component of the solar wind magnetic field is always greater than $-3 \mathrm{nT}$. Our model uses the ideal MHD approximation and consequently, it is not valid for strong southward solar wind magnetic fields, which favor reconnection and a subsequent departure from an ideal MHD situation. The corresponding error can be estimated by the earthward shift of the magnetopause due to the southward magnetic fields using the magnetopause model by Shue et al. (1998). For a solar wind magnetic field of $-3 \mathrm{nT}$, the magnetopause is about $0.2 R_{\mathrm{E}}$ closer to the Earth than computed with Eq. (22). It seems natural to assume a similar error for the bow shock distance. A further model error can occur due to the zeroth-order approximation by choosing $N_{\mathrm{S}}=0$ of the reduced model. This model error for the analytical solution compared to a more accurate second-order solution was estimated to be about $0.2 R_{\mathrm{E}}$ using the data of the magnetosheath of 24 August 2008 by Nabert et al. (2013). An error of $0.2 R_{\mathrm{E}}$ in the determination of the bow shock distance for typical solar wind conditions at Earth is related to an error of less than $6 \%$ in the calculation of the 
Table 1. Bow shock locations close to the subsolar point and the corresponding solar wind conditions observed by THC. The $z$ component of solar wind magnetic field is $B_{z, \mathrm{SW}}$ and the magnetic field magnitude in the $y z$ plane is $B_{t, \mathrm{SW}}=\left(B_{y, \mathrm{SW}}^{2}+B_{z, \mathrm{SW}}^{2}\right)^{0.5}$. The computed dipole moments are also presented.

\begin{tabular}{lrrrrrrrrr}
\hline $\begin{array}{l}\text { Date } \\
\text { (dd.m.) }\end{array}$ & $\begin{array}{r}\text { Time } \\
(\mathrm{UT})\end{array}$ & $\begin{array}{r}N_{\mathrm{SW}} \\
\left(1 \mathrm{~cm}^{-3}\right)\end{array}$ & $\begin{array}{r}v_{\mathrm{SW}} \\
\left(\mathrm{km} \mathrm{s}^{-1}\right)\end{array}$ & $\begin{array}{r}B_{z, \mathrm{SW}} \\
(\mathrm{nT})\end{array}$ & $\begin{array}{r}B_{t, \mathrm{SW}} \\
(\mathrm{nT})\end{array}$ & $\begin{array}{r}\Delta x_{\mathrm{SC} / \mathrm{BS}} \\
\left(R_{\mathrm{E}}\right)\end{array}$ & $\begin{array}{r}y_{\mathrm{SC} / \mathrm{BS}} \\
\left(R_{\mathrm{E}}\right)\end{array}$ & $\begin{array}{r}z_{\mathrm{SC} / \mathrm{BS}} \\
\left(R_{\mathrm{E}}\right)\end{array}$ & $\begin{array}{r}M / 10^{15} \\
\left(\mathrm{Tm}^{3}\right)\end{array}$ \\
\hline 24.8. & $00: 29$ & 7.61 & 310.4 & 1.11 & 1.74 & 12.59 & -5.52 & -2.27 & -7.53 \\
25.8. & $22: 41$ & 6.81 & 267.8 & 0.89 & 1.72 & 13.64 & -4.82 & -2.41 & -7.25 \\
27.8. & $22: 20$ & 8.04 & 272.6 & -1.41 & 3.58 & 13.41 & -4.43 & -2.10 & -6.94 \\
29.8. & $21: 06$ & 6.92 & 324.1 & -2.93 & 3.99 & 14.37 & -3.56 & -2.62 & -9.05 \\
31.8. & $21: 49$ & 6.98 & 334.3 & -2.47 & 2.60 & 13.63 & -3.73 & -2.02 & -8.48 \\
02.9. (a) & $19: 48$ & 6.96 & 250.1 & -1.94 & 1.98 & 15.22 & -2.40 & -1.74 & -7.92 \\
02.9. (b) & $20: 58$ & 7.43 & 276.7 & 0.93 & 2.32 & 14.17 & -2.81 & -2.57 & -8.23 \\
04.9. (a) & $21: 02$ & 2.05 & 571.5 & 1.55 & 4.10 & 14.06 & -2.33 & -1.68 & -7.52 \\
04.9. (b) & $21: 23$ & 2.05 & 562.1 & 1.50 & 4.26 & 13.76 & -2.46 & -1.62 & -6.87 \\
06.9. (a) & $21: 26$ & 4.02 & 546.9 & -3.05 & 3.78 & 13.50 & -2.20 & -1.45 & -9.42 \\
06.9. (b) & $22: 30$ & 3.93 & 560.1 & 2.92 & 3.15 & 12.47 & -2.46 & -1.49 & -7.78 \\
\hline
\end{tabular}

dipole moment $M$ according to Eq. (27). Typical solar wind conditions at the Earth are a solar wind ion particle density of about $N_{\mathrm{SW}}=7 \mathrm{~cm}^{-3}$, a velocity $v_{\mathrm{SW}}=430 \mathrm{~km} \mathrm{~s}^{-1}$, a solar wind magnetic field $B_{\mathrm{SW}}=6 \mathrm{nT}$, and an ion temperature $T_{\mathrm{SW}}=8 \times 10^{4} \mathrm{~K}$ (Slavin and Holzer, 1981).

Additionally, errors of estimating the dipole moment can occur due to a bow shock motion caused by varying solar wind conditions or data errors. The subsolar bow shock distance to the Earth's center for typical solar wind conditions at Earth is about $13 R_{\mathrm{E}}$. A variation in the solar wind density of about $20 \%$ compared to typical solar wind conditions leads to a variation in the bow shock location of about $0.3 R_{\mathrm{E}}$. This corresponds to a relative error of about $10 \%$ for the dipole moment $M$ computed by Eq. (27). Further, a variation in the solar wind velocity of about $20 \%$ corresponds to an error of about $20 \%$ for the estimated dipole moment $M$. Errors due to solar wind variations are usually statistical errors which cancel out if a mean value of a large sample size is considered in contrast to model errors, which can be systematic. The errors considered here are consistent with the estimations of $M$ using a single bow shock transition as presented in Table 1 . Further, the mean value of these estimations is closer to the true value of the Earth's dipole moment than the individual values as expected.

The method presented takes bow shock locations into account. In contrast to magnetopause observations, the solar wind conditions can be determined at bow shock crossings, even for single-spacecraft missions. The method provides a valid estimator for the planetary dipole moment, in which a larger sample size might reduce the statistical error further.

\subsection{Using magnetosheath data}

The previous considerations took into account the location of the bow shock only. From a statistical point of view, it seems advantageous to include more data points on an orbit for the estimation of the dipole moment. If the solar wind conditions during the magnetosheath crossings of THC are known, each data point within the magnetosheath can be used to estimate dipole moment $M$. However, the error of the estimation will depend on the level of knowledge of the solar wind conditions. Such an approach is very suitable for two-spacecraft missions such as BepiColombo. One spacecraft crosses the interaction region, while the other spacecraft observes the solar wind.

A model of the interaction relates the observations within the interaction region together with the solar wind data to the planetary magnetic field. Here, we use the reduced MHD model with the approximation $N_{\mathrm{S}}=0$ presented in Sect. 2 . Similar to Nabert et al. (2015), a cost function can be introduced to quantify the misfit between model solution for any choice of $M$ and observations. The planetary magnetic field parameter $M$ in the model is modified until the cost function is minimized. Then, the model predictions fit to the physical quantities measured by a spacecraft within the interaction region.

On the orbit of THC across the magnetosheath, the plasma's mass density $\rho_{\mathrm{SC}, m}$, the $x$ component of the velocity $v_{\mathrm{SC}, m}$, the $z$ component of the magnetic field $B_{\mathrm{SC}, m}$, and the gas pressure $p_{\mathrm{SC}, m}$ are measured in GSM coordinates at locations $\left(x_{\mathrm{SC}, m}, y_{\mathrm{SC}, m}, z_{\mathrm{SC}, m}\right)$ labeled with the position in$\operatorname{dex} m$. The corresponding solar wind conditions at the subsolar point for the mass density $\rho_{\mathrm{SW}, m}$, the velocity $v_{\mathrm{SW}, m}$, the $z$ component of the magnetic field $B_{\mathrm{SW}, m}$, and the pressure $p_{\mathrm{SW}, m}$ at Earth can be determined by OMNI solar wind data (http://omniweb.gsfc.nasa.gov/; King and Papitashvili, 2005) representing measurements from a second spacecraft. Analogously to the previous considerations using bow shock locations, the data in GSM coordinates are transferred into the model coordinate system with the $x$ axis aligned to the solar wind velocity vector. 
The physical quantities $\rho, v_{x}, p$, and $B_{z}$ are constant for $\widetilde{x}=$ const as seen in Eqs. (14)-(17). Thus, a location beside the $x$ axis corresponds to a position on the $x$ axis along the paraboloid geometry with the same values of the physical quantities. Consequently, a coordinate transformation can be introduced which relates the location of a data point beside the $x$ axis to a location on the $x$ axis of the model. Therefore, the magnetosheath geometry needs to be taken into account according to Eq. (13). Together with the analytical expressions of the curvature parameters, a spacecraft's location in the model coordinates $x_{\mathrm{SC}}, y_{\mathrm{SC}}, z_{\mathrm{SC}}$ is related to a location $\tilde{x}_{\mathrm{SC}}$ on the $x$ axis by

$$
\begin{aligned}
x_{\mathrm{SC}} & =\tilde{x}_{\mathrm{SC}}+\left(c_{\mathrm{BS}, y}+\Delta c_{y} \frac{\tilde{x}_{\mathrm{SC}}}{x_{\mathrm{MS}}}\right) y_{\mathrm{SC}}^{2} \\
& +\left(c_{\mathrm{BS}, z}+\Delta c_{z} \frac{\tilde{x}_{\mathrm{SC}}}{x_{\mathrm{MS}}}\right) z_{\mathrm{SC}}^{2} .
\end{aligned}
$$

Measured spacecraft data at the transformed spacecraft coordinate $\widetilde{x}_{\mathrm{SC}, m}$ of the mass density $\rho_{\mathrm{SC}, m}$, the velocity $v_{\mathrm{SC}, m}$, the magnetic field $B_{\mathrm{SC}, m}$, and the pressure $p_{\mathrm{SC}, m}$ can be compared to the model solution at locations $\widetilde{x}=\widetilde{x}_{\mathrm{SC}, m}$.

The solution of the model (14)-(17) is found by solving the differential equations (18)-(21) with the corresponding solar wind conditions and an assumption of the dipole moment $M$. A cost function is introduced to quantify the misfit of data and model solution. Using the method of least-squares regression analysis, we consider a cost function which includes the THEMIS plasma (McFadden et al., 2008) as well as the magnetic field (Auster et al., 2008) data:

$$
\begin{aligned}
K & =\sum_{m=1}^{M_{\mathrm{data}}}\left(\frac{\rho_{0}\left(\widetilde{x}_{\mathrm{SC}, m}\right)-\rho_{\mathrm{SC}, m}}{\rho_{\mathrm{SC}, m}}\right)^{2} \\
& +\left(\frac{v_{x 0}\left(\widetilde{x}_{\mathrm{SC}, m}\right)-v_{\mathrm{SC}, m}}{v_{\mathrm{SC}, m}}\right)^{2}+\left(\frac{B_{z 0}\left(\widetilde{x}_{\mathrm{SC}, m}\right)-B_{\mathrm{SC}, m}}{B_{\mathrm{SC}, m}}\right)^{2}
\end{aligned}
$$

where $M_{\text {data }}$ denotes the number of data points in the magnetosheath. The gas pressure does not contribute to this cost function because it is a second-order moment of the velocity distribution function which is difficult to determine precisely from spacecraft data.

The cost function depends on the model solution, which is a function of the dipole moment $M$. The cost function is calculated on a grid in the range $-12 \times 10^{15} \mathrm{Tm}^{3} \leq M \leq$ $-6 \times 10^{15} \mathrm{Tm}^{3}$. The grid space between two values of $M$ is taken to be $0.004 \times 10^{15} \mathrm{Tm}^{3}$. The minimum value of the cost function determines the estimated dipole moment. A value for the dipole moment is estimated from the magnetosheath data close to the $x$ axis separately for each orbit of THC between 24 August and 6 September 2008. As an example, the magnetosheath data on 31 August 2008 in the model coordinates is presented in Fig. 1. We choose a 5 min time resolution for the OMNI as well as THC spacecraft data which is in accord with the quasi-stationary approximation used in the reduced MHD model. Note that $5 \mathrm{~min}$ is the order of the

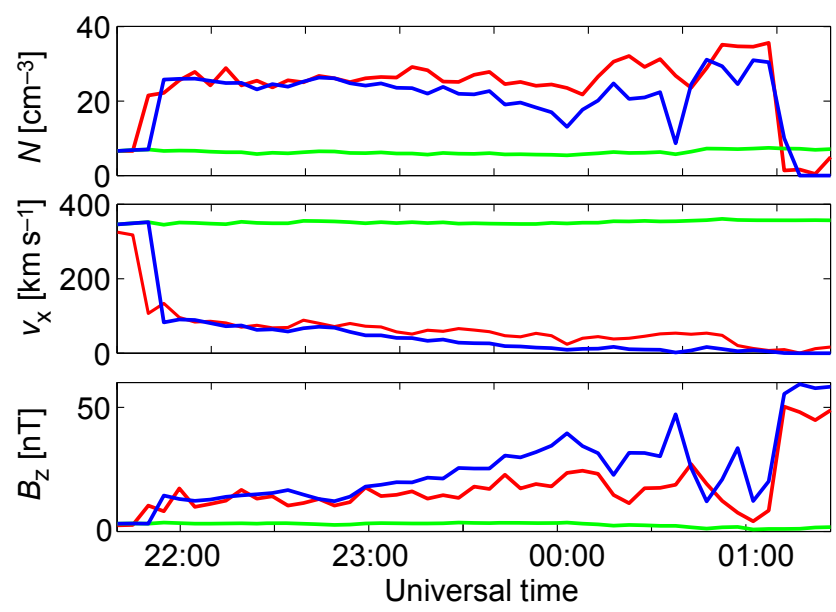

Figure 1. Magnetosheath spacecraft data on 31 August 2008 (red) and adjusted model results (blue) which determined the dipole moment to $M$ using the time-dependent OMNI solar wind data (green) presented in model coordinates.

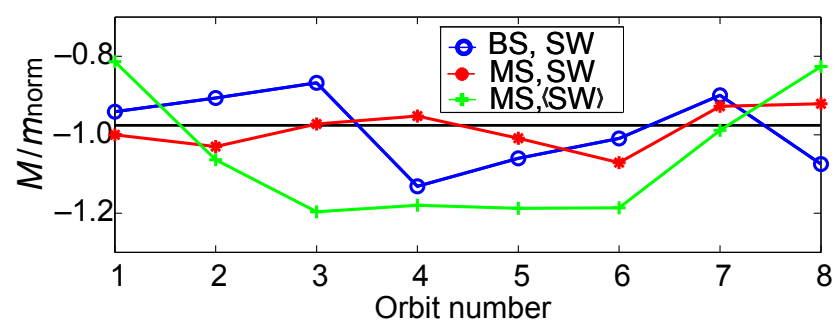

Figure 2. Estimated dipole moments using THC data at orbits from $24.8,25.8,27.8,29.8,31.8,2.9,4.9$, and 6.9 , labeled by orbit numbers 1 to 8 . The dipole moment was estimated by THC bow shock observations together with pre-shock solar wind conditions (blue), by THC magnetosheath data using the time-dependent OMNI solar wind observations, and by THC magnetosheath data with average solar wind conditions (green). The $z$ component of the Earth's dipole moment is depicted as a black line. Dipole moments are normalized to $m_{\text {norm }}=8.0 \times 10^{15} \mathrm{Tm}^{3}$.

solar wind transit time across the subsolar magnetosheath. The time-varying OMNI solar wind data are used as boundary conditions. The reduced magnetosheath model solution is adjusted to the magnetosheath data via minimization of cost function (32), which determines the dipole moment $M$. The corresponding adjusted reduced MHD model solution on 31 August 2008 is also depicted in Fig. 1. The eight estimated dipole moments from the eight magnetosheath crossings are shown in Fig. 2. Each estimated dipole moment differs not more than $13 \%$ from the value of the Earth's dipole moment. The mean value of the eight computed dipole moments is $M=-7.9 \times 10^{15} \mathrm{Tm}^{3}$ with a standard deviation of $0.4 \times 10^{15} \mathrm{Tm}^{3}$. The error is less than $4 \%$ with respect to the $z$ component and less than $2 \%$ with respect to the magnitude of the Earth's dipole moment. 


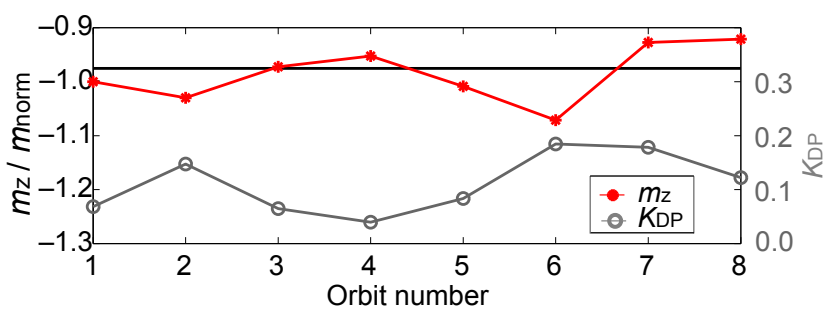

Figure 3. Estimated dipole moments (red) using cost function (32) and the value of the cost function at its minimum normalized to the number of data points of the respective magnetosheath transition (grey). The dipole moments are normalized to $m_{\text {norm }}=8.0 \times$ $10^{15} \mathrm{Tm}^{3}$.

The value of the estimated dipole moment using the entire magnetosheath data seems comparable to the estimate using bow shock location information. However, the standard deviation, related to the statistical error, is halved. The estimated dipole moments taking only bow shock locations into account are also displayed in Fig. 2. No correlation between the results of the two different methods is apparent; the correlation coefficient is 0.13 . Thus, one can assume different sources of errors for the two methods. For example, the estimations with the bow shock locations use solar wind conditions close to the subsolar point of the shock. Instead, the OMNI solar wind data take spacecraft data far away from the bow shock to estimate the conditions at Earth. The latter approach requires a model to transfer the solar wind conditions to the Earth which is more error-prone. However, using the complete time interval of magnetosheath data instead of only the bow shock location, more data are taken into account, so that statistical errors might be reduced.

Additionally to the estimated dipole moments, the value of the cost function (32) normalized to the number of data points $K_{\mathrm{DP}}:=K / M_{\text {data }}$ is considered in Fig. 3. The value of $K_{\mathrm{DP}}$ is in the range of 0.04 and 0.2 , which gives a mean deviation between data and model solution. Smaller values of the normalized cost function correspond to a better agreement between data and model solution. Values of the cost function below 0.1 , i.e., orbit numbers $1,3,4$, and 5 , correspond to estimators close to the true value. Consequently, it might be suitable to give more emphasis to estimates which show a better agreement of model solution and data.

To allow for better comparison of the two different methods, the approach using magnetosheath data is modified to take only measured bow shock locations $\Delta x_{\mathrm{BS}}$ together with the OMNI solar wind data into account. Then, cost function (32) is replaced by a new function,

$K=\left(\Delta x_{\mathrm{BS}}-\Delta x_{\mathrm{BS}, \mathrm{M}}\right)^{2}$,

where $\Delta x_{\mathrm{BS}, \mathrm{M}}$ denotes the subsolar bow shock location of the model solution corresponding to a certain dipole moment $M$. Minimizing this cost function determines the dipole moment $M$ for any solar wind conditions and measured bow shock location. The solar wind conditions using OMNI data can be determined at all shock transitions of THC considered in Table 1. The solar wind data are determined as a mean value of $10 \mathrm{~min}$ around a shock crossing. The dipole moments for all 11 shock transitions are calculated and multiple shock crossings on an orbit are summed to an average value. Thus, eight dipole moment estimators are obtained with a mean value of $M=-8.2 \times 10^{15} \mathrm{Tm}^{3}$ with a standard deviation of $1.2 \times 10^{15} \mathrm{Tm}^{3}$. This result has a significantly increased statistical error compared to the previous result using the entire magnetosheath data. The error is reduced by taking the entire magnetosheath data into account instead of only bow shock locations.

For single-spacecraft missions, the solar wind conditions during a magnetosheath transition are usually not well known and average values need to be assumed. To investigate the advantage of time-dependent considerations, the Earth dipole moment is estimated using the mean values of the solar wind conditions of all transitions considered here. These average conditions of all eight magnetosheath transitions are for the ion particle density $<N_{\mathrm{SW}}>=6.65 \mathrm{~cm}^{-3}$, the velocity $\left\langle v_{\mathrm{SW}}\right\rangle=382.2 \mathrm{~km} \mathrm{~s}^{-1}$, and the magnetic field $<$ $B_{\mathrm{SW}}>=2.84 \mathrm{nT}$. Using these average values as the boundary condition of the reduced MHD model, dipole moments $M$ can be estimated from the eight magnetosheath crossings once again. The results are also presented in Fig. 2. The mean value of the dipole moment from the eight values is $M=-8.4 \times 10^{15} \mathrm{Tm}^{3}$ with a standard deviation of $1.3 \times 10^{15} \mathrm{Tm}^{3}$. This corresponds to an error of about $10 \%$ with respect to the Earth's dipole moment.

The use of average solar wind conditions leads to a result that is worse compared to the use of time-dependent solar wind conditions. The dipole moment is significantly overestimated and standard deviation is 3 times larger. The reason is the nonlinear dependence of the MHD model solution on the solar wind conditions. Thus, for a precise estimation of the planetary magnetic field from spacecraft data obtained within a region strongly influenced by the interaction with the solar wind, it is an advantage to include the actual solar wind conditions instead of using average conditions.

\section{Conclusions}

We examined two different methods to estimate the planetary magnetic dipole field using a reduced MHD model to take the solar wind interaction into account. The methods presented were investigated in preparation for the analysis and interpretation of measurements from the BepiColombo mission to Mercury (Benkhoff et al., 2010). The results show that the precision of the estimated dipole field is much better if the actual solar wind conditions are considered instead of average values. Thus, we expect more accurate estimates of Mercury's magnetic field using BepiColombo data than previous approaches can provide. Further, the statistical error 
can be reduced by taking more orbits into account. A good estimation can be made even for single-spacecraft missions by considering bow shock location information. In contrast to magnetopause locations, the actual solar wind conditions at the bow shock can be determined using the pre-shock solar wind data. Here, this leads to an error of less than $4 \%$ for eight magnetosheath transitions considered with respect to the true value. Taking not only the bow shock location information but also data from the entire magnetosheath into account leads to similar results. However, the statistical error is significantly reduced. In summary, considering timedependent solar wind information as well as data of the entire magnetosheath gives the best estimator for the planetary dipole moment with smallest statistical error.

In this study, we used a simple reduced MHD model of the interaction. In a next subsequent step, more data need to be included to reduce the statistical error. Therefore, the reduced MHD model, which is valid close to the $x$ axis of the model, needs to be replaced by an MHD model of the entire magnetosheath. Furthermore, the estimation of higher-degree planetary moments such as a quadrupole moment should be considered.

Data availability. Data from the THEMIS mission are publicly available and can be obtained from http://themis.ssl.berkeley.edu/ data/themis (Angelopoulos, 2008) from the University of California Berkeley. The OMNI solar wind data from NASA are publicly available and can be obtained from http://spdf.gsfc.nasa.gov/pub/ data/omni (King and Papitashvili, 2005).

Competing interests. The authors declare that they have no conflict of interest.

Acknowledgements. This work was financially supported by the German Ministerium für Wirtschaft und Technologie and the Deutsches Zentrum für Luft- und Raumfahrt under contracts $50 \mathrm{OC} 1403$ and 50QW1501. We acknowledge NASA contract NAS5-02099 and V. Angelopoulos for use of data from the THEMIS Mission. Specifically: C. W. Carlson and J. P. McFadden for use of ESA data. We acknowledge use of NASA/GSFC's Space Physics Data Facility's OMNIWeb service, and OMNI data.

The topical editor, E. Roussos, thanks V. M. Vasyliunas and one anonymous referee for help in evaluating this paper.

\section{References}

Alexeev, I. I., Belenkaya, E. S., Slavin, J. A., Korth, H., Anderson, B. J., Baker, D. N., Boardsen, S. A., Johnson, C. L., Purucker, M. E., Sarantos, M., and Solomon, S. C.: Mercury's magnetospheric magnetic field after the first two MESSENGER flybys, Icarus, 209, 23-39, doi:10.1016/j.icarus.2010.01.024, 2010.

Angelopoulos, V.: The THEMIS mission, Space Sci. Rev., 141, 534, doi:10.1007/s11214-008-9336-1, 2008.
Auster, H. U., Glassmeier, K. H., Magnes, W., Aydogar, O., Baumjohann, W., Constantinescu, D., Fischer, D., Fornacon, K. H., Georgescu, E., Harvey, P., Hillenmaier, O., Kroth, R., Ludlam, M., Narita, Y., Nakamura, R., Okrafka, K., Plaschke, F., Richter, I., Schwarzl, H., Stoll, B., Valavanoglou, A., and Wiedemann, M.: The THEMIS Fluxgate Magnetometer, Space Sci. Rev., 141, 235-264, doi:10.1007/s11214-008-9365-9, 2008.

Benkhoff, J., van Casteren, J., Hayakawa, H., Fujimoto, M., Laakso, H., Novara, M., Ferri, P., Middleton, H. R., and Ziethe, R.: BepiColombo - Comprehensive exploration of Mercury: Mission overview and science goals, Planet. Space Sci., 58, 2-20, doi:10.1016/j.pss.2009.09.020, 2010.

Christensen, U. R.: A deep dynamo generating Mercury's magnetic field, Natur, 444, 1056-1058, doi:10.1038/nature05342, 2006.

Finlay, C. C., Maus, S., Beggan, C. D., Bondar, T. N., Chambodut, A., Chernova, T. A., Chulliat, A., Golovkov, V. P., Hamilton, B., Hamoudi, M., Holme, R., Hulot, G., Kuang, W., Langlais, B., Lesur, V., Lowes, F. J., Lühr, H., MacMillan, S., Mandea, M., McLean, S., Manoj, C., Menvielle, M., Michaelis, I., Olsen, N., Rauberg, J., Rother, M., Sabaka, T. J., Tangborn, A., TøffnerClausen, L., Thébault, E., Thomson, A. W. P., Wardinski, I., Wei, Z., and Zvereva, T. I.: International Geomagnetic Reference Field: the eleventh generation, Geophys. J. Int., 183, 1216-1230, doi:10.1111/j.1365-246X.2010.04804.x, 2010.

Gauss, C. F.: Allgemeine Theorie des Erdmagnetismus, in: Resultate aus den Beobachtungen des Magnetischen Vereins im Jahre 1838, edited by: Gauss, C. F. and Weber, W., 1-59, Göttinger Magnetischer Verein, Leipzig, 1839.

Glassmeier, K.-H. and Tsurutani, B. T.: Carl Friedrich Gauss General Theory of Terrestrial Magnetism - a revised translation of the German text, Hist. Geo Space. Sci., 5, 11-62, doi:10.5194/hgss-5-11-2014, 2014.

Glassmeier, K.-H., Auster, H.-U., and Motschmann, U.: A feedback dynamo generating Mercury's magnetic field, Geophys. Res. Lett., 34, L22201, doi:10.1029/2007GL031662, 2007.

Heimpel, M. H., Aurnou, J. M., Al-Shamali, F. M., and Gomez Perez, N.: A numerical study of dynamo action as a function of spherical shell geometry, Earth Planet. Sc. Lett., 236, 542-557, doi:10.1016/j.eps1.2005.04.032, 2005.

Heyner, D., Wicht, J., Gómez-Pérez, N., Schmitt, D., Auster, H.-U., and Glassmeier, K.-H.: Evidence from Numerical Experiments for a Feedback Dynamo Generating Mercury's Magnetic Field, Science, 334, 1690-1693, doi:10.1126/science.1207290, 2011.

Johnson, C. L., Purucker, M. E., Korth, H., Anderson, B. J., Winslow, R. M., Al Asad, M. M. H., Slavin, J. A., Alexeev, I. I., Phillips, R. J., Zuber, M. T., and Solomon, S. C.: MESSENGER observations of Mercury's magnetic field structure, J. Geophys. Res.-Planet., 117, E00L14, doi:10.1029/2012JE004217, 2012.

King, J. H. and Papitashvili, N. E.: Solar wind spatial scales in and comparisons of hourly Wind and ACE plasma and magnetic field data, J. Geophys. Res., 110, A02104, doi:10.1029/2004JA010649, 2005.

Kivelson, M. G. and Russell, C. T.: Introduction to Space Physics, Cambridge University Press, Cambridge, 1995.

Korth, H., Anderson, B. J., Acuña, M. H., Slavin, J. A., Tsyganenko, N. A., Solomon, S. C., and McNutt, R. L.: Determination of the properties of Mercury's magnetic field by the MESSENGER mission, Planet. Space Sci., 52, 733-746, doi:10.1016/j.pss.2003.12.008, 2004. 
Korth, H., Tsyganenko, N. A., Johnson, C. L., Philpott, L. C., Anderson, B. J., Al Asad, M. M., Solomon, S. C., and McNutt, R. L.: Modular model for Mercury's magnetospheric magnetic field confined within the average observed magnetopause, J. Geophys. Res.-Space, 120, 4503-4518, doi:10.1002/2015JA021022, 2015.

McFadden, J. P., Carlson, C. W., Larson, D., Ludlam, M., Abiad, R., Elliott, B., Turin, P., Marckwordt, M., and Angelopoulos, V.: The THEMIS ESA Plasma Instrument and In-flight Calibration, Space Sci. Rev., 141, 277-302, doi:10.1007/s11214-008-9440-2, 2008.

Mead, G. D. and Beard, D. B.: Shape of the Geomagnetic Field Solar Wind Boundary, J. Geophys. Res., 69, 1169-1179, doi:10.1029/JZ069i007p01169, 1964.

Nabert, C., Glassmeier, K.-H., and Plaschke, F.: A new method for solving the MHD equations in the magnetosheath, Ann. Geophys., 31, 419-437, doi:10.5194/angeo-31-419-2013, 2013.

Nabert, C., Othmer, C., and Glassmeier, K.-H.: Solar wind reconstruction from magnetosheath data using an adjoint approach, Ann. Geophys., 33, 1513-1524, doi:10.5194/angeo-33-15132015, 2015.

Olsen, N., Glassmeier, K.-H., and Jia, X.: Separation of the Magnetic Field into External and Internal Parts, Space Sci. Rev., 152, 135-157, doi:10.1007/s11214-009-9563-0, 2010.

Petrinec, S. M. and Russell, C. T.: Hydrodynamic and MHD Equations across the Bow Shock and Along the Surfaces of Planetary Obstacles, Space Sci. Rev., 79, 757-791, doi:10.1023/A:1004938724300, 1997.

Raines, J. M., Slavin, J. A., Zurbuchen, T. H., Gloeckler, G., Anderson, B. J., Baker, D. N., Korth, H., Krimigis, S. M., and McNutt, R. L.: MESSENGER observations of the plasma environment near Mercury, Planet. Space Sci., 59, 2004-2015, doi:10.1016/j.pss.2011.02.004, 2011.

Raines, J. M., Gershman, D. J., Zurbuchen, T. H., Sarantos, M., Slavin, J. A., Gilbert, J. a., Korth, H., Anderson, B. J., Gloeckler, G., Krimigis, S. M., Baker, D. N., McNutt, R. L., and Solomon, S. C.: Distribution and compositional variations of plasma ions in Mercury's space environment: The first three Mercury years of MESSENGER observations, J. Geophys. Res.-Space, 118, 1604-1619, doi:10.1029/2012JA018073, 2013.

Shue, J.-H., Song, P., Russell, C. T., Steinberg, J. T., Chao, J. K., Zastenker, G., Vaisberg, O. L., Kokubun, S., Singer, H. J., Detman, T. R., and Kawano, H.: Magnetopause location under extreme solar wind conditions, J. Geophys. Res., 103, 17691-17700, doi:10.1029/98JA01103, 1998.
Siscoe, G. L.: Solar system magnetohydrodynamics, in: SolarTerrestrial Physics: Principles and Theoretical Foundations, edited by: Carovillano, R. L. and Forbes, J. M., 11-100, D. Reidel Publishing Company, Dordrecht, doi:10.1007/978-94-0097194-3_2, 1983.

Slavin, J. A. and Holzer, R. E.: Solar wind flow about the terrestrial planets. I - Modeling bow shock position and shape, J. Geophys. Res., 86, 11401-11418, doi:10.1029/JA086iA13p11401, 1981.

Solomon, S. C., McNutt, R. L., Gold, R. E., Acuña, M. H., Baker, D. N., Boynton, W. V., Chapman, C. R., Cheng, A. F., Gloeckler, G., Head, III, J. W., Krimigis, S. M., McClintock, W. E., Murchie, S. L., Peale, S. J., Phillips, R. J., Robinson, M. S., Slavin, J. A., Smith, D. E., Strom, R. G., Trombka, J. I., and Zuber, M. T.: The MESSENGER mission to Mercury: scientific objectives and implementation, Planet. Space Sci., 49, 1445-1465, doi:10.1016/S0032-0633(01)00085-X, 2001.

Stanley, S., Bloxham, J., Hutchison, W. E., and Zuber, M. T.: Thin shell dynamo models consistent with Mercury's weak observed magnetic field, Earth Planet. Sc. Lett., 234, 27-38, doi:10.1016/j.epsl.2005.02.040, 2005.

Tsyganenko, N. A.: Modeling the Earth's magnetospheric magnetic field confined within a realistic magnetopause, J. Geophys. Res., 100, 5599-5612, doi:10.1029/94JA03193, 1995.

Tsyganenko, N. A.: Effects of the solar wind conditions in the global magnetospheric configurations as deduced from databased field models, in: Proceedings of 3rd Int. Conf. on Substorms (ICS-3), edited by: Rolfe, E. J. and Kaldeich, B., 181185, European Space Agency, Paris, 1996.

Wicht J., Heyner D.: Mercury's magnetic field in the messenger era, in: Planetray Geodesy and Remote Sensing, edited by: Shuanggen J., CRC Press, London, 223-262, 2014.

Winslow, R. M., Anderson, B. J., Johnson, C. L., Slavin, J. A., Korth, H., Purucker, M. E., Baker, D. N., and Solomon, S. C.: Mercury's magnetopause and bow shock from MESSENGER Magnetometer observations, J. Geophys. Res.-Space, 118, 2213 2227, doi:10.1002/jgra.50237, 2013. 